\title{
Multimodal imaging of an Archer 3 retinal racemose hemangioma
}

\section{Estudio multimodal de hemangioma racemoso retiniano Archer 3}

\author{
Romana García-Gil*, Anselmo Feliciano-Sánchez, Lucía Ramos-González and Rafael Martínez-Costa
}

Ophthalmology Service, Eye Tumor Unit, Medical and Surgical Retina Section, Hospital Universitario y Politécnico La Fe, Valencia, Spain
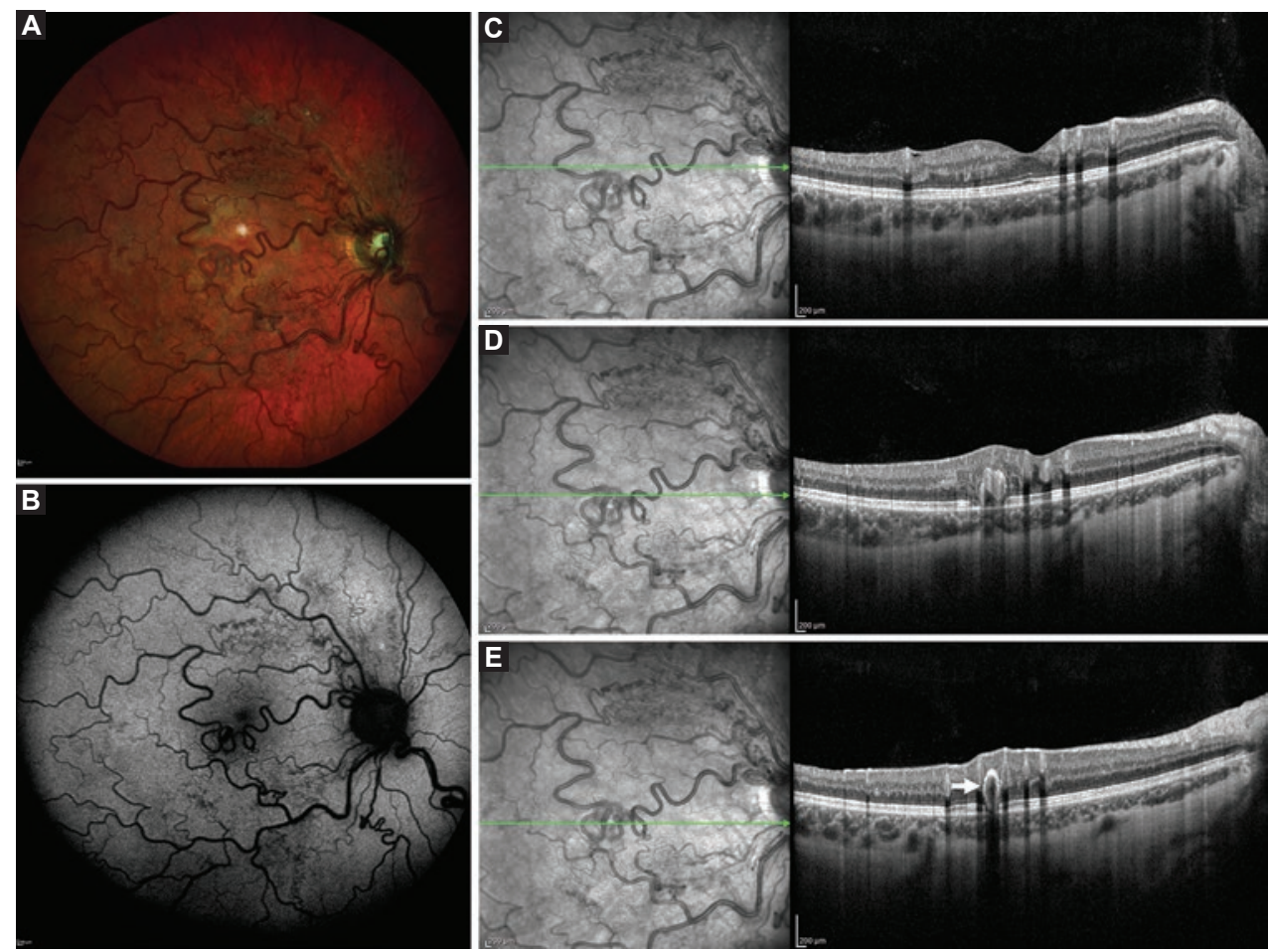

Figure 1. A: Multicolored fundus image. Dilated and tortuous retinal vessels are observed derived from the optic disc and extending to the retinal periphery as a result of an arteriovenous communication. B: Short-wave autofluorescence. A hypo-autofluorescent vascular tree preserving the foveolar area is identified with greater definition; C, D and E: OCT. A conserved foveolar profile (C) is observed, with irregularities in the internal and external retina, coinciding with the vascular malformation, that causes a posterior shadow (D); also, the vascular lumen ( $\mathbf{E}$, arrow) and choroidal thinning can be observed.

Date of reception: 04-07-2019 C.P. 46026 , Valencia, Spain E-mail: romygg9@ hotmail.com
Date of acceptance: 30-07-2019 DOI: 10.24875/RMOE.M20000099
Available online: 01-03-2020 Rev Mex Oftalmol (Eng). 2020;94(2):85-86

www.rmo.com.mx 2604-1731/@ 2019 Sociedad Mexicana de Oftalmología. Published by Permanyer. This is an open access article under the CC BY-NC-ND license (http://creativecommons.org/licenses/by-nc-nd/4.0/). 
We present the case of an asymptomatic 18-year-old female with a visual acuity of 1 in both eyes, with a casual finding in the right eye of a racemose hemangioma. This is a retinal arteriovenous malformation of congenital, non-inherited origin, classified as a sporadic phakomatosis (Fig. 1).

Racemose hemangioma of the retina is associated in $30 \%$ of cases with cerebral arteriovenous malformations, constituting Wyburn-Mason syndrome. In our case, the patient had no systemic vascular abnormalities or ocular complications.

\section{Conflicts of interest}

The authors declare no conflicts of interest.

\section{Ethical disclosures}

Protection of human and animal subjects. The authors declare that the procedures followed were in accordance with the regulations of the relevant clinical research ethics committee and with those of the Code of Ethics of the World Medical Association (Declaration of Helsinki).

Confidentiality of data. The authors declare that they have followed the protocols of their work center on the publication of patient data.

Right to privacy and informed consent. The authors have obtained the written informed consent of the patients or subjects mentioned in the article. The corresponding author is in possession of this document. 